\title{
The role of spatial working memory in inhibition of return: Evidence from divided attention tasks
}

\author{
ALAN D. CASTEL and JAY PRATT \\ University of Toronto, Toronto, Ontario, Canada \\ and \\ FERGUS I. M. CRAIK \\ Rotman Research Institute, Baycrest Centre for Geriatric Care, and \\ University of Toronto, Toronto, Ontario, Canada
}

\begin{abstract}
Inhibition of return (IOR) refers to a bias against returning attention to a location that has been recently attended. In the present experiments, we examined the role of working memory in IOR by introducing secondary tasks (in the temporal interval between the cue and the target) that involved a working memory component. When the secondary task was nonspatial in nature (monitoring odd digits or adding digits), IOR was present, although overall reaction times were greater in the presence of the secondary task. When the task involved a spatial working memory load (remembering the directionality of arrows or the orientation of objects), IOR was eliminated. However, when the participants had incentive to process the directionality of an arrow but did not have to use any memory system, IOR persisted at peripheral locations. Overall, the results suggest that IOR is partially mediated by a spatial working memory system.
\end{abstract}

The ability to search the visual environment in order to locate certain objects is a critical component of the visual system. People need to conduct searches of the visual environment because the visual system cannot fully process all of its input (Wolfe, 1994). One way to ensure that visual search is efficient is to limit attention's returning to previously searched locations. Posner and Cohen (1984) demonstrated that if attention is captured at a cued peripheral location and then moved to a different location, the time to detect the presence of a target is delayed if the target appears at the previously cued (i.e., attended) location. This effect has been referred to as inhibition of return (IOR), a label that captures the original notion that attention is inhibited from returning to previously searched locations (e.g., Posner \& Cohen, 1984; Posner, Rafal, Choate, \& Vaughan, 1985). Although the attentional explanation has received considerable support over time (e.g., Berlucchi, Chelazzi, \& Tassinari, 2000; Tipper, Weaver, Jerreat, \& Burak, 1994), motor-based explanations (e.g., Rafal, Calabresi, Brennan, \& Sciolto, 1989; Taylor \& Klein, 1998) and combinations of motor

This research was supported by Natural Sciences and Engineering Research Council of Canada (NSERC) grants to J.P. and F.I.M.C. and by a Sigma Xi Grant-in-Aid of Research and an NSERC postgraduate scholarship to A.D.C. We thank Ray Klein, David Shore, Yuji Takeda, Jeremy Wolfe, and Richard Wright for helpful comments and suggestions regarding this manuscript and Meira Lewis and Tara McAuley for help with data collection. Correspondence regarding this paper should be addressed to A. D. Castel or J. Pratt, Department of Psychology, 100 St. George Street, Toronto, ON, M5S 3G3 Canada (e-mail: alan@ psych.utoronto.ca or pratt@psych.utoronto.ca). and attentional explanations (e.g., Kingstone \& Pratt, 1999; Taylor \& Klein, 2000) have also been proposed, as well as the notion that IOR occurs as a result of spatial indexes that serve as location "pointers" that guide visual search by constraining the search set (Wright \& Richard, 1998, 2000).

Although a considerable amount of research has elucidated many facets of IOR, the processes that determine and maintain inhibition at cued locations, or objects, are still not known. Presumably, some processes associated with the visual system "tags" cued locations or objects, which subsequently biases shifts of attention and/or motor actions away from these peripheral locations. Since IOR lasts as long as 3,000 msec (e.g., Vaughan, 1984), some memory process must play a role in maintaining and updating the information regarding cued stimuli and spatial locations, allowing the visual system to carry out an efficient search of the environment. Furthermore, this memory process must be flexible enough to be updated, because IOR has been found for cued objects that have moved to novel (i.e., uncued) spatial locations (Tipper, Driver, \& Weaver, 1991; Tipper et al., 1994). One likely possibility is that an active working memory system is involved, similar to the one first proposed by Baddeley and Hitch (1974; Baddeley, 1986), which records which locations or objects have been previously attended. This working memory system includes an articulatory store and spatial stores, both of which maintain and update information that is currently being processed.

As was noted before, IOR lasts a relatively long time and stays with moving objects, suggesting that IOR is not 
due solely to low-level visual processes. Moreover, studies in which IOR at multiple cued locations has been examined have strongly suggested that there is a memory component in IOR. In their initial study on this issue, Pratt and Abrams (1995) found IOR only at the last cued location. However, they compared the IOR generated by a single cue with the IOR generated by two successive cues, using a display that contained only two locations. As was noted by Tipper, Weaver, and Watson (1996), a lack of IOR for the first two cues may have been found because the system does allow for inhibition at all possible target locations. Subsequent studies in which a greater number of potential cue/target locations than of cues (e.g., three successive cues distributed among four possible locations) has been used have shown IOR at more than the last cued location (e.g., Abrams \& Pratt, 1996; Danziger, Kingstone, \& Snyder, 1998; Snyder \& Kingstone, 2000; Tipper et al., 1996). Similarly, Wright and Richard (1998, 2000) have suggested that IOR involves some sort of spatial indexing such that multiple "tags" at previously attended locations lead to IOR and that this allows cued locations to be encoded and maintained over time. It is possible that this is similar to a system suggested by Pylyshyn (1989), who proposed a resource-limited mechanism, called the finger of instantiation (abbreviated as FINST), for individuating or indexing visual features in a visual field. FINST is capable of mapping locations on the basis of the retina location, such that FINST can keep pointing to the "same" feature cluster as the cluster moves across the retina. Taken together, these studies strongly suggest that IOR involves some type of working memory process that allows multiple spatial locations to be inhibited over a relatively long period of time.

Further support for the notion that memory is involved in IOR comes from separate sets of studies that have concluded (1) that memory plays a role in visual searches (Gilchrist \& Harvey, 2000; Shore \& Klein, 2000; but see Horowitz \& Wolfe, 1998, 2001, for counterarguments) and (2) that IOR plays a role in visual search tasks (Klein, 1988; Müller, \& von Mühlenen, 2000; Shore \& Klein, 2000; Takeda \& Yagi, 2000). Indeed, IOR has been found with searches in which the number of locations that are inhibited ranges from 8 through 12 (Ogawa, Takeda, \& Yagi, 2002; Peterson, Kramer, Wang, Irwin, \& McCarley, 2001) to as many as 20 (Takeda, in press). Given that both memory and IOR have been implicated in improving the efficiency of visual search, it seems likely that the two processes interact with each other. Indeed, similar to the findings from the multiple sequential cue studies, these visual search studies suggest there is working memory involvement in IOR, given that inhibition must be maintained at several previously examined locations over the duration of the search.

Perhaps the best way to examine whether working memory is involved in IOR is to use the dual-task paradigm. It is generally agreed that working memory requires attentional resources to function effectively (e.g., Cowan, 1995, 1999). It therefore follows that the intro- duction of a secondary task that also requires attentional resources will reduce working memory performance. Furthermore, if IOR depends on working memory, the simultaneous performance of a secondary task should reduce or eliminate the presence of IOR. To date, the effect of introducing a secondary task in an IOR paradigm has been examined in only one experiment that we know of. Maylor (1985) conducted an experiment in which the notion that a demanding secondary task would disrupt IOR was examined. In this series of experiments, in addition to responding to the presence or absence of a target in one of two peripheral locations (a detection task), participants also engaged simultaneously in one of four secondary tasks. These secondary tasks involved the pursuit eye tracking of a slowly moving dot inside the central box that was present at the fixation area, and the four conditions consisted of the dots moving horizontally, the dots moving vertically, horizontal to vertical motion after the cue, and vertical to horizontal motion after the cue. While carrying out this task at fixation, the participants made detection responses (the primary task) to either a cued or an uncued target that appeared in one of two peripheral locations. The results showed no IOR to previously cued locations when there was no earlier attentional facilitation, suggesting that IOR is dependent on prior orienting. However, these secondary tasks did not involve any memory component, so it is unclear whether or not a secondary task that involves a working memory component would also reduce the effects of IOR. Furthermore, it is well known that attention and eye movements are intimately linked (e.g., Deubel \& Schneider, 1996; Shepherd, Findlay, \& Hockey, 1986). Thus, secondary tasks that involve pursuit eye movements are also likely to influence the spatial allocation of attention, making it difficult to interpret the findings from Maylor's experiment.

In the present study, we sought to examine the role of working memory in IOR. If IOR involves working memory, it should be possible to affect IOR with a divided attention manipulation that involves a working memory load. In order to study this, the allocation of resources was manipulated by presenting to-be-remembered items at fixation during the interval between the presentations of the cue and the target. In the first experiment, a verbal working memory load was used in which three digits were presented between the cue and the target and the participants reported the number of odd digits that appeared, after responding to the target (Experiment 1). In order to examine the effects of a spatial working memory load, in the next two experiments, the digits at fixation were replaced by a sequence of upward and downward pointing arrows, and the participants were required either to monitor and remember the direction of the arrows (Experiment 2) or to ignore the arrows (Experiment 3). In Experiment 4, we examined whether an intervening shift of attention in response to an arrow can eliminate IOR, and Experiment 5 replicated and extended the findings from Experiment 2 with a different kind of spatial working memory task. 


\section{EXPERIMENT 1}

The aim of Experiment 1 was to examine the effect of introducing a divided attention task on IOR. If IOR requires memory and attentional resources similar to those required by the secondary task, this should reduce the magnitude of IOR. The display consisted of two different target locations and a central fixation area. One of the locations was briefly cued, and the target then appeared at either the cued or the uncued location. In the divided attention condition, a digit-monitoring task was used such that the participants had to identify the number of odd digits that were presented after the cue and before the target. This type of task requires the recruitment of a working memory system to maintain and update the numbers presented during the interval between the presentations of the cue and the target. If IOR is dependent on a general working memory system, the introduction of a working memory task should reduce or eliminate IOR.

\section{Method}

Participants. Ten undergraduate students from the University of Toronto participated in the experiment in return for course credit. All had normal or corrected-to-normal vision and were naive with regard to the purpose of the experiment. The participants did not participate in any other experiment in the laboratory and were new to the general procedure.

Apparatus and Procedure. The experiment took place in a dimly lit sound-attenuate $d$ room. The participants were seated $44 \mathrm{~cm}$ in front of a computer monitor. The viewing distance was held constant with the use of an adjustable head-/chinrest, and the computer keyboard was placed directly in front of the participants. The sequence of events in Experiment 1 is shown in the left panel of Figure 1, although all of the stimuli were presented in white on a black background. The initial display was presented for $500 \mathrm{msec}$, and consisted of two placeholder boxes located on the horizontal meridian to the left and right of the fixation point. The boxes were centered $5^{\circ}$ from the fixation point and were $1^{\circ}$ square. One of the boxes was then cued by outlining the perimeter of the box for $100 \mathrm{msec}$. Then, in the divided attention condition, a random number was presented at the central fixation area for $300 \mathrm{msec}$, followed by a $50-\mathrm{msec}$ delay, a second random number for $300 \mathrm{msec}$,

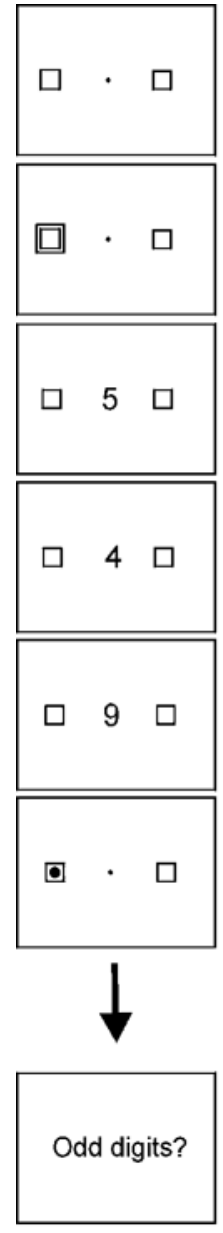

Exp 1
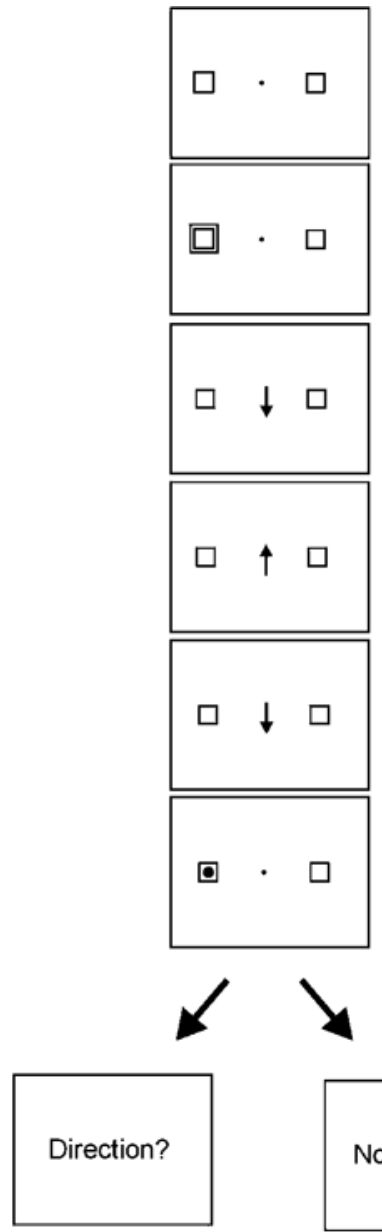

$\operatorname{Exp} 2$
TIME

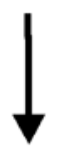

Figure 1. The sequence of events in noncatch trials in Experiment 1 (left), Experiment 2 (middle), and Experiment 3 (right). 
a second 50-msec delay, and a third random number for $300 \mathrm{msec}$. The random numbers ranged from 1 to 9 . In the control condition, the participants were not presented with digits but were presented with three visual events. After the cue in the control condition, the fixation point remained on for $300 \mathrm{msec}$, followed by a $50-\mathrm{msec}$ delay, a fixation point twice the size of the previous point for $300 \mathrm{msec}$, a 50-msec delay, and the original fixation point for $300 \mathrm{msec}$. Following this sequence of fixation events, in the control and the divided attention conditions, a target circle $\left(0.7^{\circ}\right)$ appeared in one of the two boxes (on $80 \%$ of the trials, whereas the remaining $20 \%$ served as catch trials in which no target was presented). The participants were asked to respond to the target as quickly and as accurately as possible by pressing the space bar (regardless of the location of the target) and to remain fixated throughout each trial. The detection target remained in view until the participant made a response or until 1,000 msec had elapsed, after which a tone sounded if the participant did not respond (on noncatch trials). In addition, the participants in the divided attention condition were also asked to determine how many odd digits had appeared between the cue and the target. After they had responded to the target, they were prompted as to how many odd digits they had seen, and they entered this number on the keyboard. The participants were told that both the detection task and the odd-digit monitoring were equally important.

Design. The divided attention condition and the control condition were blocked, and both were completed in a single 1-h session. The order of the conditions was counterbalanced across participants. Each condition consisted of 120 trials, with cues and targets being equally likely to occur at the left and the right locations. The participants were given a short break between the conditions.

\section{Results and Discussion}

The mean reaction times (RTs) for correct trials are shown in the left panel of Figure 2. Trials were elimi- nated if an error occurred either in responding to the target or in making the odd-digits response. The data were submitted to a 2 (trial type: cued or uncued) $\times 2$ (condition: divided attention or control) analysis of variance (ANOVA). There was a main effect of trial type $[F(1,9)=$ 17.2, $\left.M S_{\mathrm{e}}=237, p<.005\right]$, indicating that RTs were slower when the target appeared at the cued location. This is the typical finding of IOR. The main effect of condition was also significant $\left[F(1,9)=17.7, M S_{\mathrm{e}}=5,860\right.$, $p<.005]$, indicating that RTs under divided attention were slower than RTs in the control condition. Importantly, the interaction between condition and trial type was not significant $[F(1,9)<1]$. The lack of a significant interaction revealed that the divided attention condition did not interfere with the presence of IOR. Indeed, IOR was present, in almost equal magnitude, in the divided attention and the control conditions. These findings indicate that, contrary to our expectations, working memory is apparently not involved in IOR.

In order to clarify the errors that can be made, we have classified errors as detection errors (responding less than $100 \mathrm{msec}$ after the presentation of the target or failing to respond within $1,000 \mathrm{msec}$ after the target has been presented), catch trial errors (responding on trials in which the target is not presented), and secondary task errors (not providing the correct answer to the question). The error rates are presented in Table 1. In order to examine any potential speed-accuracy tradeoffs in the cued and uncued trials, the detection errors were sub-

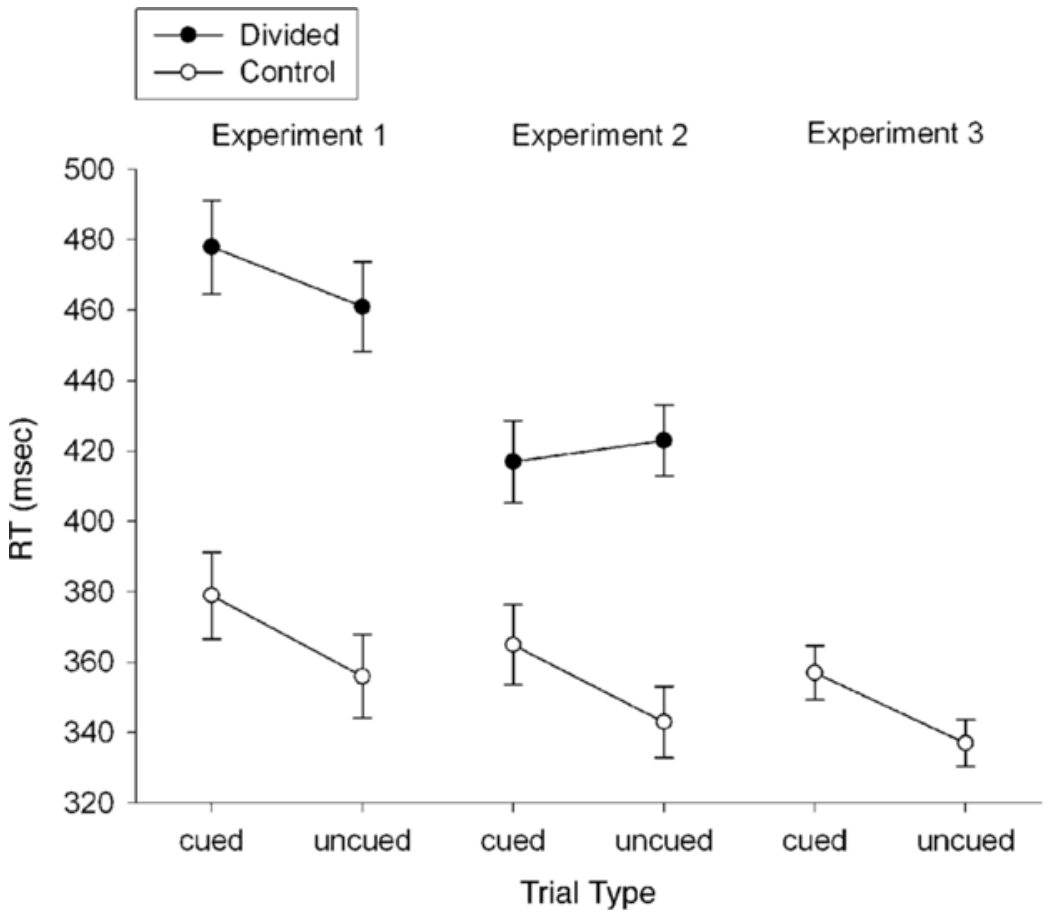

Figure 2. Mean reaction times (RTs) for correct trials in the divided attention conditions and the control conditions in Experiments 1, 2, and 3. Error bars indicate standard errors of the mean. 
Table 1

Mean Percentages of Detection Errors and Secondary Task Errors (When Applicable) for Cued and Uncued Locations in All Five Experiments

\begin{tabular}{lccc}
\hline \multicolumn{1}{c}{ Condition } & \multicolumn{2}{c}{ Location } & \\
\cline { 2 - 3 } & Cued & Uncued & Mean \\
\hline Control & 0.33 & 0.33 & \\
Divided attention & 4.33 & 2.50 & 3.33 \\
Secondary task & 3.00 & 4.17 & 3.59 \\
& Experiment 2 & & \\
Control & 0.83 & 0.83 & 0.83 \\
Divided attention & 5.00 & 4.44 & 4.72 \\
Secondary task & 3.61 & 2.78 & 3.19 \\
& Experiment 3 & & \\
Control & 0 & 0 & 0 \\
& Experiment 4 & & \\
Peripheral cue & 0.59 & 0.30 & 0.45 \\
Arrow cue & 1.07 & 0.37 & 0.72 \\
& Experiment 5 & & \\
Divided attention & 3.67 & 4.83 & 4.28 \\
Secondary task & 8.33 & 9.67 & 9.00 \\
\hline
\end{tabular}

Note-Detection errors are classified as occurring in either the control condition or the divided attention condition, whereas secondary task errors refer to errors made on the secondary task in the divided attention condition.

mitted to a 2 (trial type: cued or uncued) $\times 2$ (condition: divided attention or control) ANOVA. There was a main effect of condition $\left[F(1,9)=9.1, M S_{\mathrm{e}}=2.89, p<.05\right.$; showing that more errors were made in the divided attention condition] but no main effect of trial type $\left[F(1,9)<2.0, M S_{\mathrm{e}}=1.50, p>.19\right]$ and no significant interaction $\left[F(1,9)<3.1, M S_{\mathrm{e}}<1, p>.11\right]$. Catch trial errors were made on $4.8 \%$ of all the catch trials in the divided attention condition and on $0.33 \%$ of all the catch trials in the control conditions, and this difference was found to be statistically significant $[t(9)=3.29, p<.01]$. In the divided attention condition, errors on the digitmonitoring task were committed on fewer than $8 \%$ of the trials, and the numbers of errors were not significantly different for cued and uncued trials $[t(9)<1.8, p>.11]$. The error rate on the secondary task suggests that it does demand a certain amount of attention but that the errors committed on this task did not differ for cued and uncued trials, ruling out any speed-accuracy tradeoffs in terms of detection RTs for cued and uncued trials.

The present findings suggest that IOR exists when participants must process numerical information in working memory. ${ }^{1}$ If IOR relies on a more spatial component of working memory, the introduction of a spatial working memory task should disrupt IOR.

\section{EXPERIMENT 2}

Experiment 2 was designed to test whether a spatial working memory task would disrupt the mechanisms that govern IOR. However, the spatial working memory task could not involve any peripheral events, since such events would cause a shift in attention and, therefore, produce a reduction in IOR. Thus, the spatial information in the secondary task had to be incorporated into an event at the central fixation location. To accomplish this, the participants in this experiment were asked to monitor three briefly presented vertical arrows to determine whether the majority of the arrows were oriented upward or downward. This task is similar to the previous divided attention tasks but involves a spatial component similar to the binary decision that was made in Experiment 1 regarding the odd digits. If the arrow secondary task uses spatial working memory and if IOR has a spatial working memory component, this secondary task should reduce IOR.

\section{Method}

Participants. Twelve new undergraduate students from the University of Toronto participated in the experiment in return for course credit. All had normal or corrected-to-normal vision and were naive with regard to the purpose of the experiment.

Apparatus and Procedure. The experiment took place in a dimly lit sound-attenuate $d$ room. The participants were seated $44 \mathrm{~cm}$ in front of a computer monitor. The viewing distance was held constant with the use of an adjustable head-/chinrest, and the computer keyboard was placed directly in front of the participants.

The sequence of events in Experiment 2 is shown in the middle panel of Figure 1. The apparatus and basic trial sequence was similar to those in Experiment 1, with the major difference being that arrows were presented between the presentation of the cue and the target. The three arrows were presented one after the other and were either oriented upward or oriented downward. The arrows were $1^{\circ}$ in height and were presented at the center fixation area. The arrows were presented for the same duration as the digits in Experiment 1. The participant's task was to identify the direction (either up or down) of the majority of the three arrows on each trial. Thus, if two arrows pointed down and one pointed up, the correct response would be down. The participants answered the question by pressing the key on the numeric keypad corresponding to an up arrow (the 8 key) or the key corresponding to a down arrow (the 2 key). As in Experiment 1, the participant was given an unlimited amount of time to answer the question, and once the participant made this response, the next trial began $800 \mathrm{msec}$ later. The control condition was identical to that in Experiment 1, with the participants being presented with three visual events (enlarging fixation point) between the presentations of the cue and the target. As in Experiment 1 , after the cue in the control condition, the fixation point remained on for $300 \mathrm{msec}$, followed by a $50-\mathrm{msec}$ delay, a fixation point twice the size of the previous point for $300 \mathrm{msec}$, a 50 -msec delay, and the original fixation point for $300 \mathrm{msec}$. The participants did not have to monitor the stimuli or make any response regarding the stimuli. In the control condition, the next trial began $800 \mathrm{msec}$ after the participant responded to the target.

\section{Results and Discussion}

The mean RTs for correct detection responses are shown in the middle panel of Figure 2. Only trials in which the participant correctly answered the question regarding the arrows were included in the analysis. The data were submitted to a 2 (trial type: cued or uncued) $\times 2$ (condition: divided attention or control) repeated measures ANOVA. The main effect of trial type was not statistically significant $\left[F(1,11)=2.28, M S_{\mathrm{e}}=336, p<.16\right]$. The main effect of condition was statistically significant $\left[F(1,11)=18.1, M S_{\mathrm{e}}=2,855, p<.005\right]$. Unlike Experiments 1 and 2, the critical interaction between condition 
and trial type was significant $\left[F(1,11)=19.5, M S_{\mathrm{e}}=113\right.$, $p<.002]$.

In the present experiment, three types of errors were possible, as outlined in Experiment 1. The detection error rates are presented in Table 1. In order to examine any possible speed-accuracy tradeoffs in the cued and uncued trials, the detection errors were submitted to a 2 (trial type: cued or uncued) $\times 2$ (condition: divided attention or control) ANOVA. There was a main effect of condition $\left[F(1,11)=5.71, M S_{\mathrm{e}}=6.96, p<.05\right.$; showing that more errors were made in the divided attention condition] but no main effect of trial type $[F(1,11)<1]$ and no significant interaction $[F(1,11)<1]$. Catch trial errors were made more often in the divided attention condition (on $3.1 \%$ of all the catch trials) than in the control condition [on $0.63 \%$ of all the catch trials; $t(11)=3.15$, $p<.01]$. In the divided attention condition, errors on the arrow-monitoring task were committed on fewer than $4 \%$ of the trials, and the number of errors was not significantly different for cued and uncued trials $[t(11)<1]$.

The finding of a significant interaction between condition and trial type, as can be seen in Figure 2, strongly suggests that IOR was not present in the divided attention condition. Thus, it appears that processing and retaining information about the arrows reduces or even eliminates the biasing of attention to uncued or novel locations. Since the participants made a decision regarding the directionality of the majority of the arrows, it is likely that they used some form of spatial working memory to process the arrows. During this processing period, information about previously attended locations decayed, was displaced, or became less accessible. This observation provides initial evidence for a spatial working memory component that guides IOR, so that when other spatial information competes for spatial working memory capacity, memory for previously attended locations becomes less accessible.

An alternative explanation for the reduction in IOR in the divided attention condition is also possible, however. It may be that the mere presence of the arrows caused the participants to orient their attention in the direction of the arrows. Evidence for such obligatory shifts has come from a recent study by Hommel, Pratt, Colzato, and Godijn (2001). If such intervening shifts of attention occurred in the interval between the peripheral cues and the peripheral targets, this might have contributed to the finding of equivalent RTs to the cued and the uncued locations. In order to investigate this possibility, a control experiment was carried out in which the arrows were present but the participants did not need to monitor or remember the directionality of the arrows (i.e., no memory component to the secondary task).

\section{EXPERIMENT 3}

Experiment 3 was designed to rule out the possibility that it was the presence of the arrows that eliminated IOR in Experiment 2 because of obligatory shifts of attention (e.g., Hommel et al., 2001). Although the arrows were never predictive of the location of the target (since they pointed either up or down), it is still possible that the participants shifted their attention according to the directionality of the arrows and that this eliminated IOR from the peripherally cued location. The present experiment used stimuli identical to those in Experiment 2, but in this case the participants did not need to remember or respond to the directionality of the arrows. If the elimination of IOR in the previous experiment was due to the spatial working memory load, and not to the mere presence of the arrows, IOR should be found in the present experiment.

\section{Method}

Participants. Six undergraduate students from the University of Toronto participated in the experiment in return for course credit. All had normal or corrected-to-normal vision and were naive with regard to the purpose of the experiment.

Apparatus and Procedure. The present experiment was identical to the divided attention condition in Experiment 2, except that the participants did not need to remember the directionality of the arrows (the participants were told that the arrows were irrelevant to the task of detecting the target). The participants responded only to the presence of the target (see the right panel of Figure 1).

\section{Results and Discussion}

The mean RTs for correct detection responses are shown in the right panel of Figure 2. Detection errors were made on fewer than $1 \%$ of the trials, which was not enough to analyze. The difference in RT to the two locations was found to be statistically significant $[t(5)=$ $2.21, p<.05]$, indicating the presence of IOR.

In contrast to the lack of IOR in Experiment 2, the finding of IOR in the present experiment suggests that it is remembering the directionality of the arrows, and not just their presence, that interferes with IOR. Thus, the results from the present experiment support the notion that the introduction of a spatial working memory load disrupts IOR but that the presence of irrelevant spatial information does not by itself interfere with IOR. However, there may have been a difference in the way in which the arrows were attended to in Experiments 2 and 3 that may have been the cause of the presence and absence of IOR. This possibility was examined in Experiment 4 .

\section{EXPERIMENT 4}

An alternative interpretation of the results found in Experiments 2 and 3 focuses on how the participants processed the central arrows. In both experiments, the arrows appeared as abrupt onsets, so it is likely that the arrows were reflexively attended to in the two experiments. However, in Experiment 2, the participants presumably also volitionally attended to the arrows, because they had to determine whether they pointed mostly up or down. This was not the case in Experiment 3, where they were told to ignore the arrows. It may be that the lack of 
IOR found with the arrows in Experiment 2 was not due to any working memory load but, rather, that volitionally attending to the arrows produced shifts of attention that interfered with the IOR generated from the peripheral cues. Because there was no need to attend to the arrows in Experiment 3, the arrows might not have produced shifts of attention, and thus there was no interference of IOR.

The present experiment was conducted to determine whether volitionally attending to a central arrow and then producing a volitional shift of attention in the direction of that arrow would interfere with IOR. In this experiment, the display consisted of four placeholder boxes equidistant from each other and from the fixation area, oriented so that two boxes were located along the horizontal meridian (as in all the of the previous experiments) and two were located in the vertical meridian. Following a peripheral cue at one of the locations in the horizontal meridian, a vertical arrow appeared at the central fixation area and remained present until the end of the trial. This arrow was predictive of where the target would appear, and the participants were made aware of this in the instructions (the target was three times more likely to appear in the location indicated by the vertical arrow than in any of the other three locations). Because the arrow is informative, the participants should shift their attention to the location indicated by the arrow. If such an intervening shift of attention interferes with IOR (it would occur after the peripheral cue and before the onset of the target), no IOR should be found. However, if spatial working memory load was the cause of the lack of IOR in Experiment 2, IOR should be found in the present experiment.

\section{Method}

Participants. Ten new undergraduate students from the University of Toronto participated in the experiment in return for course credit. All had normal or corrected-to-normal vision and were naive with regard to the purpose of the experiment.

Apparatus and Procedure. The sequence of events in Experiment 4 is shown in Figure 3. The initial display was presented for $750 \mathrm{msec}$ and consisted of four placeholder boxes ( $1^{\circ}$ square), with two boxes located on the horizontal meridian and two boxes located on the vertical meridian. Each box was equidistant $\left(5^{\circ}\right)$ from the central fixation point, which was $0.1^{\circ}$ in diameter. One of the peripheral boxes located on the horizontal meridian (left or right) was cued by outlining the perimeter of the box for $100 \mathrm{msec}$ in white. After a 50-msec delay, a vertical arrow ( $1^{\circ}$ in height), oriented either up or down, was presented at the central fixation area and remained present for the duration of the trial. After an $850-\mathrm{msec}$ delay, a target circle $\left(0.7^{\circ}\right)$ appeared in one of the four placeholder boxes on $80 \%$ of all the trials ( $20 \%$ of the trials were catch trials). The target was three times more likely to appear in the location that the arrow pointed toward (either the top or the bottom box on the vertical meridian), and the participants were told of this prior to the experiment. Thus, trials in which the arrow predicted the target (arrow-cued trials), as well as trials in which the arrow did not predict the target (arrow-uncued trials), were intermixed with trials in which the target was presented in the same location as the peripheral cue (peripheral-cued trials), as well as trials in which the target appeared in the uncued peripheral location (peripheral-uncued trials). On all the trials, the participants were asked to respond to the

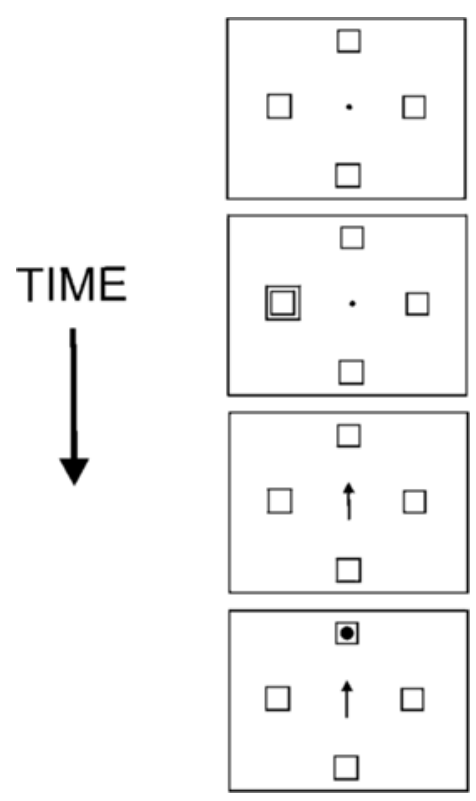

Figure 3. The sequence of events in noncatch trials in Experiment 4 . In any given trial, the central arrow can appear pointing either up or down, and the peripheral cues can appear at either the left or the right location. The target is three times more likely to appear in the vertical location indicated by the arrow.

target as quickly and as accurately as possible by pressing the space bar (regardless of the location of the target) and were told not to respond if the target did not appear. The participants were asked to remain fixated on the central fixation area throughout each trial. The next trial began 1,000 $\mathrm{msec}$ after the participants made their response or, on catch trials, 1,000 $\mathrm{msec}$ after the display disappeared. There were 270 trials in the experiment, and the participants took a short break after sets of 90 trials.

\section{Results and Discussion}

The mean RTs for correct detection responses are shown in Figure 4. Trials in which the arrow indicated the location of upcoming targets in the vertical plane are termed arrow-cued trials, and trials in which the target appeared in the same location as the peripheral cue in the horizontal plane are termed peripheral-cued trials.

The data were submitted to a 2 (condition: target appearing in the vertical plane or target appearing in the horizontal plane) $\times 2$ (trial type: arrow cued or uncued or peripheral cued or uncued, depending on the trial) repeated measures ANOVA. The main effect of condition was statistically significant $\left[F(1,9)=15.86, M S_{\mathrm{e}}=514\right.$, $p<.01]$. The main effect of trial type was not statistically significant [because one effect was a cuing effect, whereas the other was an inhibitory effect; $F(1,9)=1.47$, $\left.M S_{\mathrm{e}}=1,123, p<.3\right]$. The interaction between condition and trial type was significant $\left[F(1,9)=24.5, M S_{\mathrm{e}}=512\right.$, $p<.01]$. As can be seen in Figure 4, RTs were faster to the arrow-cued location than to the arrow-uncued location, indicating that the participants did shift their attention in accordance with the arrows. Importantly, RTs 


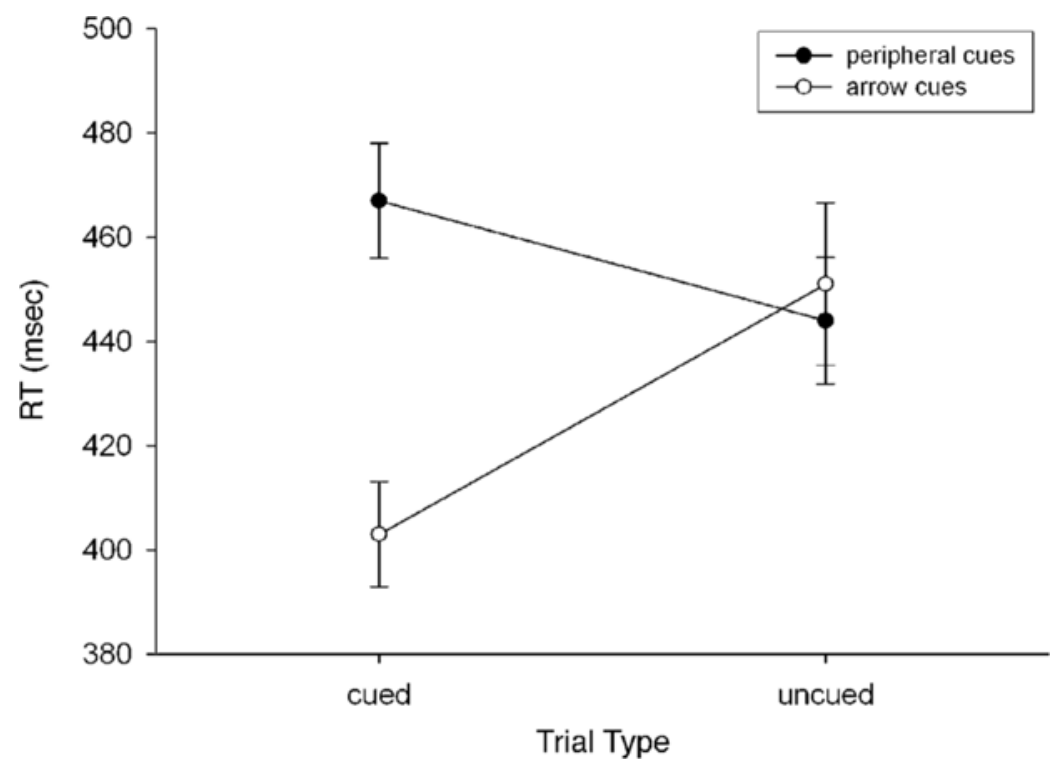

Figure 4. Mean reaction times (RTs) for correct trials in Experiment 4. Error bars indicate standard errors of the mean.

were slower to the peripherally cued locations than to the peripherally uncued locations, indicating that IOR was present despite an intervening shift of attention. Followup $t$ tests confirmed these observations, with RTs for the uncued position being shorter than those for the cued position for the peripheral cues $[t(9)=2.29, p<.05]$ and RTs for the position in which the arrow pointed at being shorter than those for the position that the arrow did not point at $[t(9)=3.25, p<.05]$. The number of detection errors are presented in Table 1; these occurred on fewer than $2 \%$ of all the trials and were not further analyzed.

The finding that IOR still exists in the presence of a predictive endogenous spatial cue that does not require working memory suggests that IOR can be found in tasks that require one to attend to and process spatial information. This is consistent with other findings reported by Berger (1994, cited in Rafal \& Henik, 1994), Berger and Henik (2000), and Berlucchi et al. (2000). Furthermore, the present study rules out an attentional set explanation in the previous studies, in which control and divided attention conditions were blocked. If this blocking procedure led to an attentional set in the divided attention condition and this is what led to the disruption of IOR, one would not expect to find IOR in the present study, in which predictive arrow cues were present in each trial, along with peripheral cues. The present results show that IOR is still observed under these circumstances, and this finding stands in contrast to those of Experiment 2, in which having to remember spatial information regarding the arrows eliminated IOR.

\section{EXPERIMENT 5}

The results of Experiments 2, 3, and 4 indicated that spatial working memory plays a critical role in IOR.
However, this conclusion was based largely on the interaction found in the arrow-monitoring memory task from the second experiment. To provide a stronger test of the conclusion, the present experiment used a different spatial working memory task. Specifically, the participants had to monitor and remember three objects (composed of two adjacent colored circles) that were presented at fixation (after the cue and before the target), in order to compare these objects with a probe object presented after the response to the peripheral target. This task had a heavy spatial component because the probe object was rotated $90^{\circ}$ clockwise from the orientation of the three memorized objects. This task was chosen because it would involve both a visual working memory component (similar to those used by Woodman, Vogel, \& Luck, 2001) and a spatial working memory component (since the participants would have to remember the relative location of the colored circles in order to carry out the rotation to allow a comparison with the probe).

\section{Method}

Participants. Ten new undergraduate students from the University of Toronto participated in the experiment in return for course credit. All had normal or corrected-to-normal vision and were naive with regard to the purpose of the experiment.

Apparatus and Procedure. The experiment took place in a dimly lit sound-attenuate $d$ room. The participants were seated $44 \mathrm{~cm}$ in front of a computer monitor. The viewing distance was held constant with the use of an adjustable head-/chinrest, and the computer keyboard was placed directly in front of the participants.

The sequence of events in Experiment 5 is shown in Figure 5. The apparatus and basic trial sequence were similar to those in Experiments 1,2 , and 3, with the major difference being that objects (as opposed to digits or arrows) were presented between the presentations of the cue and the target. The three objects were composed of two adjacent circles $\left(0.4^{\circ}\right.$ in both height and width, making the entire horizontal object $0.4^{\circ}$ in height and $0.8^{\circ}$ in width) and 

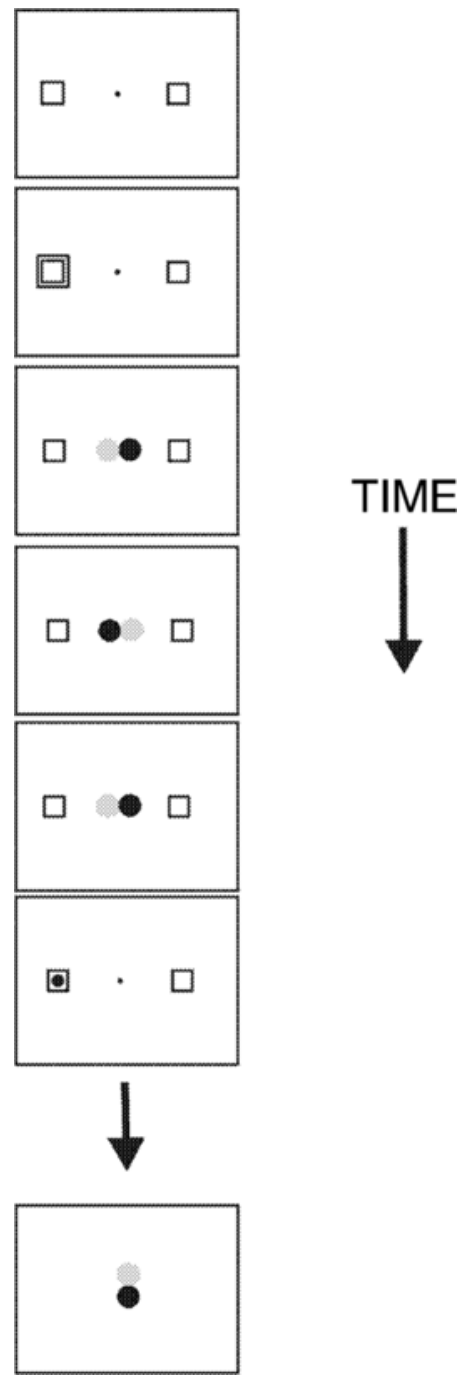

Figure 5. The sequence of events in noncatch trials in Experiment 5. The figure is not to scale (see the Method section for exact dimensions).

were oriented either with a red $\left(17 \mathrm{~cd} / \mathrm{m}^{2}\right)$ circle on the left and a green $\left(22.5 \mathrm{~cd} / \mathrm{m}^{2}\right)$ circle on the right or vice versa (i.e., there were no objects that had two green circles or two red circles). The three objects were randomly presented one after the other $(300 \mathrm{msec}$ each, with a gap of $50 \mathrm{msec}$ between presentations) and were presented at the center fixation area. The objects were presented for the same duration as the digits and arrows in Experiments 1, 2, and 3.

As with Experiment 2, the participants were told that performances on the detection task and the object-matching task were equally important. The detection task was the same as that in the previous experiments, and the participants were instructed to press the space bar as soon as they detected the target and to withhold this response if the target did not appear (i.e., on catch trials). They were also told to monitor and remember the objects that were presented at fixation, in order to compare the objects that appeared on the majority of presentations with the probe target (which they were told was rotated $90^{\circ}$ clockwise relative to the presented objects), which appeared after the participants had responded to the target (or after 1,200 msec on catch trials). For example, if two objects appeared with a red circle on the left and a green circle on the right and the probe object was a red circle above a green circle, the participants should respond same. The participants answered the question by pressing the 8 key on the numeric keypad for same or the 2 key for different. As in Experiments 1 and 2, the participant was given an unlimited amount of time to answer the question, and once the participant made this response, the next trial began $800 \mathrm{msec}$ later. As in the previous experiments, the participants were told that they should maintain fixation at the center during each trial and that they should not make any eye movements. The participants completed 120 trails in a single session, which took place in a single 1 -h session. As before, catch trials occurred on $20 \%$ of all the trials.

\section{Results and Discussion}

The mean RTs for correct detection responses were calculated using the trials in which the participant correctly answered the question regarding the objects. The mean RTs are displayed in Figure 6, and were analyzed using a two-tailed $t$ test, which did not show a reliable difference in RTs for targets at cued locations relative to targets at uncued locations $[t(9)<1.5, p>.18]$. This finding of no IOR (in fact, the nonsignificant effect was in the opposite direction) strongly suggests that monitoring and remembering the spatial component of objects presented at fixation disrupts IOR. The error rates in the present experiment are displayed in Table 1. In order to examine for speed-accuracy tradeoffs, the percentages of errors made in cued and uncued conditions were compared and were not found to be statistically different $[t(9)<1.8, p>.05]$. Thus, as was found in Experiment 2 , when participants must process and remember spatial information in working memory, IOR is disrupted, suggesting that IOR relies on a visual spatial working memory mechanism to inhibit previously cued locations.

\section{GENERAL DISCUSSION}

The results from the present study provide new insights into the processes involved in IOR. The first experiment showed that IOR exists even when numerical information must be processed and maintained after the presentation of a cue. Thus, it appears that the IOR task and the digit tasks recruited different cognitive or neural resources; even though RTs were greater in the divided attention condition, IOR was still present. In Experiment 2, when spatial information had to be processed and remembered, the inhibition associated with previously cued locations was disrupted. Experiments 3 and 4 showed that this disruption of IOR occurred only when the spatial information had to be fully processed and held in memory. Experiment 5 demonstrated that IOR is disrupted when participants must remember orientation information regarding an object in spatial working memory. The present series of experiments extends the initial finding by Maylor (1985) by having participants conduct working memory tasks that do not involve shifts of attention or pursuit eye movements and that allow for the specific examination of the attentional and memory components of IOR. 


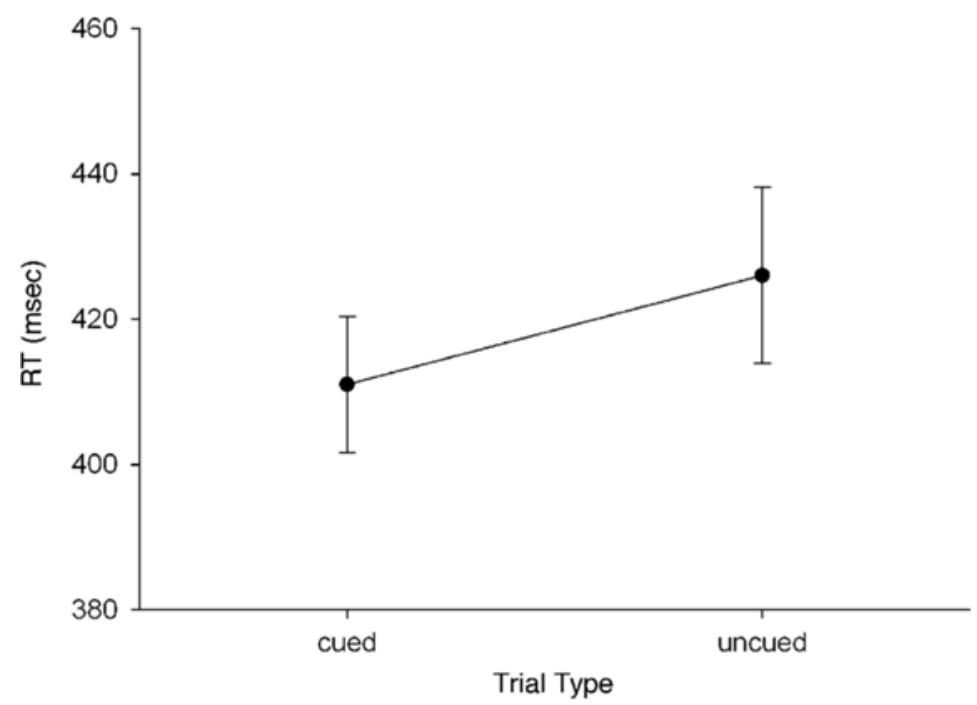

Figure 6. Mean reaction times (RTs) for correct trials in Experiment 5. Error bars indicate standard errors of the mean.

There are at least two possible explanations for the disruption of IOR when the intervening arrows and objects were processed and held in working memory in Experiments 2 and 5. One explanation is that the processing of the peripheral cue and the information presented at fixation may share common mechanisms, leading to similarity-based interference, a notion that was tested in Experiment 4. Along these lines, Berger (1994, cited in Rafal \& Henik, 1994) and Berger and Henik (2000) conducted experiments using a central arrow cue (which indicated the most likely target location) and a peripheral cue (which was uninformative) and found IOR that resulted from the peripheral cues. Notably, this pattern of results was not affected by the order in which the two cues were presented. Moreover, Berlucchi et al. (2000) reported similar findings when the central cue consisted of a number indicating the most likely target location. Given these findings and the finding from Experiment 5, it seems unlikely that the processing of peripheral and central spatial cues in close temporal proximity would be sufficient to disrupt IOR.

A second possible explanation for the disruption of IOR, and the more likely one on the basis of the present experiments, focuses on the role of visual spatial working memory in IOR. This explanation suggests that the inhibited location is held in a form of visual spatial working memory, such that intervening tasks using the same working memory processes disrupt the trace of the inhibited location. Recall that in Experiments 3 and 4, IOR was not disrupted by the mere appearance of the intervening arrows but was disrupted only when the spatial information about the arrows had to be remembered, as in Experiment 2. This pattern of results was also found when location information regarding objects had to be remembered in order to compare them with a rotated probe object. These observations raise the question of the capacity of the spatial working memory system that appears to mediate IOR. There is evidence that spatial working memory has a relatively limited capacity, perhaps capable of processing only single units of spatial information (Baddeley \& Logie, 1999). In the present case, it may be that the spatial information associated with arrows forms a unit of information that interferes with the processing of the unit of spatial information associated with the peripheral cue. It is important to note that in the present series of experiments, two different kinds of spatial working memory task both resulted in the disruption of IOR, providing converging evidence suggesting that a form of visual spatial working memory is involved in IOR. The idea that spatial working memory processes are involved in IOR has been alluded to by Klein (2000), who noted that IOR is unlikely to be due to inhibition in the superior colliculus (SC) itself (on the basis of work by Dorris, Taylor, Klein, \& Munoz, 1999) but, rather, may be due to reduced inputs to the SC from the parietal cortex. Klein suggested the parietal cortex as the possible locus of IOR because of its role in the reflexive orienting of attention, manual responding, and spatial working memory. Thus, the SC may best be viewed as an endpoint for IOR processing, and IOR may be viewed as reflecting the operation of several cortical processes, and not strictly as a subcortical, reflexive phenomenon.

The notion that spatial working memory plays a role in IOR has implications for a variety of issues regarding the deployment of attention across the visual field. One such issue concerns the number of peripherally cued locations that can be inhibited. Initially, Pratt and Abrams (1995) failed to find evidence for IOR at multiple locations. However, this appears to have been due to their method, which used only two possible cue/target loca- 
tions. Subsequent work (Abrams \& Pratt, 1996; Tipper et al., 1996) in which four possible cue/target locations were used showed IOR at more than one location. More recently, Snyder and Kingstone (2000; see also Danziger et al., 1998) showed that IOR may be found at as many as six or seven locations, consistent with the notion that IOR improves the efficiency of visual searches. Both studies found that IOR declines with increasing numbers of cues (i.e., the largest amount of IOR at the last cued location, the smallest amount of IOR at the initially cued location). Surprisingly, it is not yet known whether the reduction in IOR is due to interference from subsequent cues or simply occurs with the passage of time. Given the limited capacity of spatial working memory, combined with the relatively brief duration of the sequence of cues, it seems likely that interference from subsequent cues is the dominant factor in reducing IOR in such experiments. The present finding is also consistent with work done by Wright and Richard $(1998,2000)$, who suggested that IOR evolves from a goal-directed inhibition procedure that encodes and tags previously attended locations in order to bias search to novel locations.

Another important consideration for the role of spatial working memory in IOR concerns the notion that IOR improves the efficiency of visual searches. Logan (1978, 1979) has shown that the maintenance of verbal working memory does not interfere with visual search, whereas Woodman et al. (2001) have shown that visual search remains efficient even when visual working memory is at capacity. However, this does not rule out a role for visual spatial working memory in visual search, and recent evidence has shown that spatial working memory load impairs visual search efficiency (Oh \& Kim, 2002). In terms of IOR and visual search, Horowitz and Wolf (2001; see also Horowitz \& Wolfe, 1998) suggested that IOR and memory play a minimal role in visual search. However, other recent studies have confirmed Klein's (1988) original finding of IOR in an attentive serial visual search task, but not in a preattentive parallel search task (Müller \& von Mühlenen, 2000; Shore \& Klein, 2000; Takeda \& Yagi, 2000). Also, IOR has been found in visual search tasks that require saccadic eye movements to find targets in complicated visual displays (Gilchrist \& Harvey, 2000; Klein \& MacInnes, 1999). Gilchrist and Harvey also suggested that the number of refixations to previously cued locations in a visual search may indicate a limited functional capacity for memory of spatial locations. This would be consistent with the results from multiple-cue experiments (e.g., Snyder \& Kingstone, 2000) and with Baddeley and Logie's (1999) suggestion that visual temporary memory may be able to hold only a single pattern. However, recent research has shown that many locations may be inhibited (as many as 20 specific locations; Takeda, in press), which may suggest a very large capacity of the spatial working memory system that underlies IOR. Taken together, these experiments provide a strong case for IOR (and spatial working memory) in visual search tasks. However, as was pointed out by Horowitz and Wolfe (2001), the presence of IOR may depend on the nature of the visual search, with IOR being less likely in searches that allow for very rapid shifts of attention.

In summary, the present series of experiments show that IOR can exist in the presence of other tasks that require memory and attention but that, when such tasks demand a form of visual spatial working memory capacity, IOR is eliminated. These results and other findings from the literature suggest that IOR may rely on a visual spatial working memory system that functions to improve the efficiency of visual searches.

\section{REFERENCES}

Aвrams, R. A., \& Pratt, J. (1996). Spatially-diffuse inhibition affects multiple locations: A reply to Tipper, Weaver, and Watson (1996). Journal of Experimental Psychology: Human Perception \& Performance, 22, 1294-1298.

BADDEley, A. D. (1986). Working memory. Oxford: Oxford University Press.

Baddeley, A. D., \& Hitch, G. J. (1974). Working memory. In G. H. Bower (Ed.), The psychology of learning and motivation: Advances in research and theory (Vol. 8, pp. 47-89). New York: Academic Press.

Baddeley, A. D., \& Logie, R. H. (1999). Working memory: The multiple-component model. In A. Miyake \& P. Shah (Eds.), Models of working memory: Mechanisms of active maintenance and executive control (pp. 28-61). New York: Cambridge University Press.

Berger, A., \& HeniK, A. (2000). The endogenous modulation of IOR is nasal-temporal asymmetric. Journal of Cognitive Neuroscience, 12, 421-428.

Berlucchi, G., Chelazzi, L., \& Tassinari, G. (2000). Volitional covert orienting to a peripheral cue does not suppress cue-induced inhibition of return. Journal of Cognitive Neuroscience, 12, 648-663.

Cowan, N. (1995). An embedded-processes model of working memory. In A. Miyake \& P. Shah (Eds.), Models of working memory: Mechanisms of active maintenance and executive control (pp. 62101). New York: Cambridge University Press.

Cowan, N. (1999). Attention and memory: An integrated framework. New York: Oxford University Press.

Danziger, S., Kingstone, A., \& SNyder, J. J. (1998). Inhibition of return to successively stimulated locations in a sequential visual search paradigm. Journal of Experimental Psychology: Human Perception \& Performance, 24, 1467-1475.

Deubel, H., \& Schneider, W. X. (1996). Saccade target selection and object recognition: Evidence for a common attentional mechanism. Vision Research, 36, 1827-1837.

Dorris, M. C., Taylor, T. L., Klein, R. M., \& Munoz, D. P. (1999). Influence of previous visual stimulus or saccade on saccadic reaction times in monkey. Journal of Neurophysiology, 81, 2429-2436.

Gilchrist, I. D., \& HarveY, M. (2000). Refixation frequency and memory mechanism in visual search. Current Biology, 10, 1209-1212.

Hommel, B., Pratt, J., Colzato, L., \& Godisn, R. (2001). Symbolic control of visual attention. Psychological Science, 12, 360-365.

Horowitz, T. S., \& Wolfe, J. M. (1998). Visual search has no memory. Nature, 394, 575-577.

Horowitz, T. S., \& Wolfe, J. M. (2001). Search for multiple targets: Remember the targets, forget the search. Perception \& Psychophysics, 63, 272-285.

Kingstone, A., \& Pratt, J. (1999). Inhibition of return is composed of attentional and oculomotor processes. Perception \& Psychophysics, 61, 1046-1054

KLEIN, R. M. (1988). Inhibitory tagging system facilitates visual search. Nature, 334, 430-431.

KLEIN, R. M. (2000). Inhibition of return. Trends in Cognitive Sciences, 4, 138-147.

KLEIN, R. M., \& MacInnes, W. J. (1999). Inhibition of return is a for- 
aging facilitator in visual search. Psychological Science, 10, $346-$ 382 .

Logan, G. D. (1978). Attention in character classification task: Evidence for the automaticity of components stages. Journal of Experimental Psychology: General, 107, 32-63.

LogAN, G. D. (1979). On the use of a concurrent memory load to measure attention and automaticity. Journal of Experimental Psychology: Human Perception \& Performance, 5, 189-207.

MAYLOR, E. A. (1985). Facilitatory and inhibitory components of orienting in visual space. In M. I. Posner \& O. S. M. Marin (Eds.), Attention and performance XI (pp. 189-204). Hillsdale, NJ: Erlbaum.

Müller, H. J., \& VON Mühlenen, A. (2000). Probing distractor inhibition in visual search: Inhibition of return. Journal of Experimental Psychology: Human Perception \& Performance, 26, 1591-1605.

OgaWA, H., Takeda, Y., \& YAGI, A. (2002). Inhibitory tagging on randomly moving objects. Psychological Science, 13, 125-129.

$\mathrm{OH}$, S.-H., \& KIM, M.-S. (2002). The role of spatial working memory in visual search efficiency. Manuscript submitted for publication.

Peterson, M. S., Kramer, A. F., Wang, R. F., Irwin, D. E., \& McCarley, J. S. (2001). Visual search has memory. Psychological Science, 12, 287-292.

Posner, M. I., \& Cohen, Y. (1984). Components of visual orienting. In H. Bouma \& D. G. Bouwhuis (Eds.), Attention and performance X: Control of language processes (pp. 531-556). Hillsdale, NJ: Erlbaum.

Posner, M. I., Rafal, R. D., Choate, L., \& Vaughan, J. (1985). Inhibition of return: Neural basis and function. Cognitive Neuropsychology, 2, 211-228.

PratT, J., \& ABRAMS, R. A. (1995). Inhibition of return to successively cued spatial locations. Journal of Experimental Psychology: Human Perception \& Performance, 21, 1343-1353.

PyLyShyn, Z (1989). The role of location indexes in spatial perception: A sketch of the FINST spatial-index model. Cognition, 32, 65-97.

Rafal, R. D., Calabresi, P. A., Brennan, C. W., \& Sciolto, T. K. (1989). Saccade preparation inhibits reorienting to recently attended locations. Journal of Experimental Psychology: Human Perception \& Performance, 15, 673-685.

RAFAL, R. [D.], \& HENIK, A. (1994). The neurology of inhibition:Integrating controlled and automatic processes. In D. Dagenbach \& T. H. Carr (Eds.), Inhibitory processes in attention, memory, and language (pp. 1-52). San Diego: Academic Press.

ShePherd, M., Findlay, J. M., \& Hockey, R. J. (1986). The relationship between eye movements and spatial attention. Quarterly Journal of Experimental Psychology, 38A, 475-491.

Shore, D. I., \& KLEIN, R. M. (2000). On the manifestations of memory in visual search. Spatial Vision, 14, 59-75.

SNYDER, J. J., \& KInGSTONE, A. (2000). Inhibition of return and visual search: How many separate loci are inhibited? Perception \& Psychophysics, 62, 452-458.
TAKedA, Y. (in press). Search for multiple targets: Evidence for memory based control of attention. Psychonomic Bulletin \& Review.

TAKedA, Y., \& YAGI, A. (2000). Inhibitory tagging in visual search can be found if search stimuli remain visible. Perception \& Psychophysics, 62, 927-934.

TAY LOR, T. L., \& KLEIN, R. M. (1998). On the causes and effects of inhibition of return. Psychonomic Bulletin \& Review, 5, 625-643.

TAYlor, T. L., \& KLEIN, R. M. (2000). Visual and motor effects in inhibition of return. Journal of Experimental Psychology: Human Perception \& Performance, 26, 1639-1656.

Tipper, S. P., Driver, J., \& Weaver, B. (1991). Object-centered inhibition of return of visual attention. Quarterly Journal of Experimental Psychology, 43A, 289-298.

Tipper, S. P., Weaver, B., Jerreat, L. M., \& Burak, A. L. (1994). Objectand environment-based inhibition of return of visual attention. Journal of Experimental Psychology: Human Perception \& Performance, 30, 478-499.

Tipper, S. P., Weaver, B., \& Watson, F. (1996). Inhibition of return to successively cued spatial locations: A commentary on Pratt and Abrams (1995). Journal of Experimental Psychology: Human Perception \& Performance, 22, 1290-1293.

VAUGHAN, J. (1984). Saccades directed at previously attended locations in space. In A. G. Gale \& F. Johnson (Eds.), Theoretical and applied aspects of eye movement research (pp. 143-150). Amsterdam: NorthHolland.

Wolfe, J. M. (1994). Guided Search 2.0: A revised model of visual search. Psychonomic Bulletin \& Review, 1, 202-238.

Woodman, G. F., Vogel, E. K., \& LucK, S. J. (2001). Visual search remains efficient when visual working memory is full. Psychological Science, 12, 219-224.

Wright, R. D., \& RichaRd, C. M. (1998). Inhibition of return in not reflexive. In R. D. Wright (Ed.), Visual attention (pp. 330-347). New York: Oxford University Press.

Wright, R. D., \& RichaRd, C. M. (2000). Location cue validity affects inhibition of return of visual processing. Vision Research, 40, 23512358.

\section{NOTE}

1. An experiment similar to Experiment 1 was also carried out using a different verbal working memory load. This experiment was identical to Experiment 1, except that the participants were asked to add the three intervening digits and report the total after responding to the peripheral target. As in Experiment 1, equal magnitudes of IOR were found in both the control and the working memory load conditions.

(Manuscript received July 9, 2002; revision accepted for publication February 24, 2003.) 